\title{
Transversely polarized Drell-Yan measurements at COMPASS
}

\author{
Bakur Parsamyan* $\dagger$ \\ CERN, University of Turin and INFN section of Turin \\ E-mail: bakur.parsamyan@cern.ch
}

\begin{abstract}
The exploration of the transverse spin structure of the nucleon by measuring spin (in)dependent azimuthal asymmetries in semi-inclusive DIS (SIDIS) and in Drell-Yan processes is one of the main objectives of the COMPASS experiment at CERN (SPS, M2 beamline). During the first phase of the experiment (2002-2011) a series of SIDIS measurements were performed, using a longitudinally polarized muon beam impinging on transversely polarized ${ }^{6} \mathrm{LiD}$ or $\mathrm{NH}_{3}$ targets. As a part of the COMPASS-II programme, in 2015 and 2018 the experiment performed Drell-Yan measurements with a $\pi^{-}$beam interacting with a transversely polarized $\mathrm{NH}_{3}$. The measurement of the Sivers and other azimuthal asymmetries at the same hard scale in polarized SIDIS and Drell-Yan provides a unique possibility to test predicted in QCD (pseudo-)universal features of transverse momentum dependent parton distribution functions.
\end{abstract}

XXVII International Workshop on Deep-Inelastic Scattering and Related Subjects - DIS2019 8-12 April, 2019

Torino, Italy

* Speaker.

${ }^{\dagger}$ On Behalf of the COMPASS collaboration 


\section{Introduction}

The TMD PDFs are universal, process-independent functions ${ }^{1}$ [1] describing longitudinal and transverse momenta distributions of partons and their correlations with nucleon and quark spins. Within Leading Order (LO) QCD parton model approach the polarized nucleon is described by six time reversal even and two time reversal odd twist-2 quark transverse momentum dependent (TMD) parton distribution functions (PDFs). Correlations between nucleon and quark spins and quark intrinsic momenta induce azimuthal modulations (asymmetries) in the cross sections of SIDIS $\left(\ell N \rightarrow \ell^{\prime} h X\right.$, semi-inclusive hadron production in deep-inelastic lepton-nucleon scattering) and of Drell-Yan (DY) process ( $h N \rightarrow \ell \bar{\ell} X$, massive lepton-pair production in hadron-nucleon collisions). Applying the TMD factorization theorems [1] allows one to express the asymmetries arising in DY and SIDIS cross sections in terms of convolutions of perturbatively calculable hard-scattering parton cross sections, hard-scale dependent TMD PDFs and (for SIDIS) parton fragmentation functions (FFs). The hard scale $Q$ in SIDIS is given by the square root of the virtuality of the photon exchanged in the DIS process and in DY by the invariant mass of the produced lepton pair.

When the polarizations of the produced leptons are summed over, the general expression for the cross section of pion induced DY lepton-pair production off a transversely polarized nucleon comprises one polar asymmetry, two unpolarized and five target transverse-spin-dependent azimuthal asymmetries (TSAs). Adopting general notations and conventions of Refs. [2, 3], the model-independent differential cross section can be written as follows:

$$
\begin{aligned}
\frac{d \sigma}{d q^{4} d \Omega} \propto \hat{\sigma}_{U}\{1+ & D_{\left[\sin 2 \theta_{C S}\right]} A_{U}^{\cos \varphi_{C S}} \cos \varphi_{C S}+D_{\left[\sin ^{2} \theta_{C S}\right]} A_{U}^{\cos 2 \varphi_{C S}} \cos 2 \varphi_{C S} \\
+ & S_{T}\left[D_{\left[1+\cos ^{2} \theta_{C S} A_{T}^{\sin \varphi_{S}} \sin \varphi_{S}\right.}\right. \\
& +D_{\left[\sin ^{2} \theta_{C S}\right]}\left(A_{T}^{\sin \left(2 \varphi_{C S}-\varphi_{S}\right)} \sin \left(2 \varphi_{C S}-\varphi_{S}\right)+A_{T}^{\sin \left(2 \varphi_{C S}+\varphi_{S}\right)} \sin \left(2 \varphi_{C S}+\varphi_{S}\right)\right) \\
& \left.\left.+D_{\left[\sin 2 \theta_{C S}\right]}\left(A_{T}^{\sin \left(\varphi_{C S}-\varphi_{S}\right)} \sin \left(\varphi_{C S}-\varphi_{S}\right)+A_{T}^{\sin \left(\varphi_{C S}+\varphi_{S}\right)} \sin \left(\varphi_{C S}+\varphi_{S}\right)\right)\right]\right\},
\end{aligned}
$$

Here, $q$ is the four-momentum of the exchanged virtual photon, $F_{U}^{1}, F_{U}^{2}$ are the polarization and azimuth-independent structure functions and $\hat{\sigma}_{U}=\left(F_{U}^{1}+F_{U}^{2}\right)\left(1+\lambda \cos ^{2} \theta_{C S}\right)$, with $\lambda$ being the polar angle asymmetry, given as $\lambda=\left(F_{U}^{1}-F_{U}^{2}\right) /\left(F_{U}^{1}+F_{U}^{2}\right)$. The subscript $(U) T$ denotes target transverse polarization (in)dependence. In analogy to SIDIS, the virtual-photon depolarization factors are defined as $D_{\left[f\left(\theta_{C S}\right)\right]}=f\left(\theta_{C S}\right) /\left(1+\lambda \cos ^{2} \theta_{C S}\right)$ with $f\left(\theta_{C S}\right)$ being equal either to $\sin 2 \theta_{C S}$, or $\sin ^{2} \theta_{C S}$, or to $1+\cos ^{2} \theta_{C S}$. The angles $\varphi_{C S}, \theta_{C S}$ and $\Omega$, the solid angle of the lepton, are defined in the Collins-Soper frame following the considerations of Refs. [2,3], and $\varphi_{S}$ is the azimuthal angle of the direction of the nucleon polarization in the target rest frame, see Fig. 1.

The asymmetries $A_{(U) T}^{w}$ in Eq. 1.1 are defined as amplitudes of a given azimuthal modulation $w=w\left(\varphi_{S}, \varphi_{C S}\right)$, divided by the spin and azimuth-independent part of the DY cross section and the corresponding depolarization factor.

One spin-independent asymmetry and three out of five TSAs enter at leading order of perturbative QCD and can be described by contributions from only twist-2 TMD PDFs. In DY leptonpair production with a transversely polarized nucleon in the initial state, the $A_{U}^{\cos 2 \varphi_{C S}}$ asymmetry

\footnotetext{
${ }^{1} \mathrm{QCD}$ generalized universality: time-reversal modified process-independence of TMD PDFs
} 
is related to the convolution of nucleon and pion Boer-Mulders TMD PDFs, $\left(h_{1}^{\perp}\right.$ and $h_{1, \pi}^{\perp}$, correspondingly). The TSA $A_{T}^{\sin \varphi_{S}}$ is related to the nucleon Sivers TMD PDFs $\left(f_{1 T}^{\perp}\right)$ convoluted with the unpolarized pion TMD PDFs $\left(f_{1, \pi}\right)$. Here, following similar SIDIS conventions, the twist-3 contribution to the Sivers TSA is neglected ${ }^{2}$. Analogously, within the twist-2 approximation of LO pQCD, $F_{U}^{2}=0$ and therefore $\lambda=1$. The other two twist-2 TSAs, $A_{T}^{\sin \left(2 \varphi_{C S}-\varphi_{S}\right)}$ and $A_{T}^{\sin \left(2 \varphi_{C S}+\varphi_{S}\right)}$, are related to convolutions of the Boer-Mulders TMD PDFs $\left(h_{1, \pi}^{\perp}\right)$ of the pion with the nucleon TMD PDFs transversity $\left(h_{1}\right)$ and pretzelosity $\left(h_{1 T}^{\perp}\right)$, respectively [2, 4]. The remaining three azimuthal asymmetries, namely $A_{U}^{\cos \varphi_{C S}}, A_{T}^{\sin \left(\varphi_{C S}-\varphi_{S}\right)}$ and $A_{T}^{\sin \left(\varphi_{C S}+\varphi_{S}\right)}$ are subleading-twist structures and are expected to vanish at $\mathrm{LO}$.

All three aforementioned twist-2 nucleon TMD PDFs related to LO DY TSAs induce analogous twist-2 TSAs in the general expression for the cross section of unpolarized-hadron production in SIDIS of leptons off transversely polarized nucleons $[2,4,5]$. The SIDIS TSAs were measured by the HERMES and COMPASS experiments, see Refs. [6, 7, 8, 9, 10].

The Sivers function [11] plays an important role among the TMD PDFs. In the TMD framework of QCD it is predicted that the two naively time-reversal odd TMD PDFs, i.e. the quark Sivers functions $f_{1 T}^{\perp}$ and Boer-Mulders functions $h_{1}^{\perp}$, have opposite sign when measured in SIDIS and in DY (or $W / Z$-boson) productions. [12]. The experimental test of this fundamental prediction, which is a direct consequence of QCD gauge invariance, is a

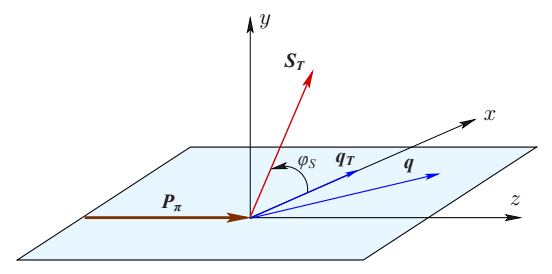
major challenge in hadron physics. In contrast to

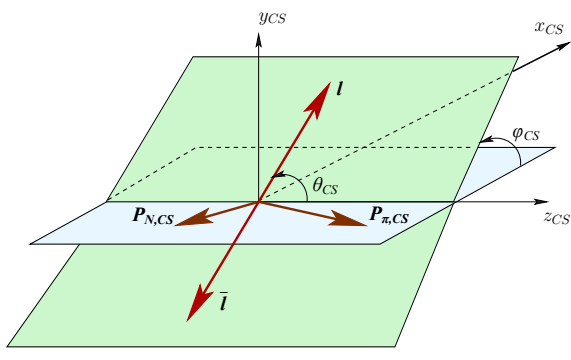

Figure 1: The target rest (top) and the Collins-Soper frames (bottom). the Sivers and Boer-Mulders functions, transversity and pretzelosity TMD PDFs are predicted to be genuinely universal, i.e. they do not change sign between SIDIS and DY [1], which is yet another fundamental QCD prediction to be explored.

Non-zero quark Sivers TMD PDFs have been extracted from SIDIS single-differential results of HERMES [8], COMPASS [6] and JLab [13] using both collinear and TMD evolution approaches $[14,15,16]$. The first measurement of the Sivers effect in $W$ and $Z$-boson production in collisions of transversely polarized protons at RHIC was reported by the STAR collaboration [17]; the hard scales of these measurements is $Q \approx 80 \mathrm{GeV} / c$ and $90 \mathrm{GeV} / c$. It is quite different from the one explored in fixed-target experiments and it is not excluded that TMD evolution effects may be sizable when describing the STAR results using Sivers TMD PDFs extracted from fixed-target SIDIS results.

The COMPASS experiment at CERN [3] has the unique capability to explore the transversespin structure of the nucleon in a similar kinematic region by two alternative experimental approaches, i.e. SIDIS and DY. This offers the opportunity of minimizing the uncertainties related

\footnotetext{
${ }^{2}$ the $F_{U T}^{2}$ structure function is assumed to be zero [3]
} 
to the TMD evolution in the comparison of the Sivers TMD PDFs when extracted from these two measurements to test the opposite-sign prediction by QCD. Recently, COMPASS published the first multi-differential results of the TSAs, which were extracted from SIDIS data at four different hard scales [9]. In order to simplify the comparison with the Drell-Yan case, one of the selected $Q^{2}$ ranges was chosen to be the same as the one selected for DY TSAs analysis.

COMPASS results for three twist-2 Drell-Yan TSAs from 2015 measurements were published in Ref. [18]. In this Letter preliminary COMPASS results obtained from the part $(\approx 50 \%)$ of the 2018 data are shown in combination with 2015 TSA measurements.

\section{Data analysis}

The analysis presented in this Letter is based on Drell-Yan data collected by COMPASS in the years 2015 and 2018. So far only part of 2018 data $(\approx 50 \%)$ has been processed and analyzed. For the measurement, the $190 \mathrm{GeV} / c \pi^{-}$beam with an average intensity of $0.6 \times 10^{8} \mathrm{~s}^{-1}$ was scattered off the COMPASS transversely polarized $\mathrm{NH}_{3}$ target. The polarized target, placed in a $0.6 \mathrm{~T}$ dipole magnet, consisted of two longitudinally aligned cylindrical cells of $55 \mathrm{~cm}$ length and $4 \mathrm{~cm}$ in diameter, separated by a $20 \mathrm{~cm}$ gap. A $240 \mathrm{~cm}$ long structure made mostly of aluminum oxyde with a tungsten core, placed downstream of the target, acted as hadron absorber and beam dump. The distribution of dimuon production vertices in the target region is shown in Fig. 2 with indicated target cell, aluminum target and tungsten core positions. The two cells were polarized vertically in opposite directions, so that data with both spin orientations were recorded simultaneously. In order to compensate for acceptance effects, the polarization was reversed approximately every two weeks. The average proton polarization was measured to be $\left\langle P_{T}\right\rangle \approx 0.73$. The dilution factor, $f$, accounting for the fraction of polarizable nucleons in the target and the migration of reconstructed events from one target cell to the other or from unpolarized medium into the cells, is estimated to be $\langle f\rangle \approx 0.18$.

The dimuon invariant mass range selected for TSA analysis is defined by the requirement $4.3 \mathrm{GeV} / c^{2}<M_{\mu \mu}<8.5 \mathrm{GeV} / c^{2}$, where the upper limit avoids the contribution of $\Upsilon$-resonances. In this range, the overall background contribution to the Drell-Yan sample is estimated to be below 4\%. The reconstructed mass spectrum of events passing all analysis requirements is shown in black in Fig. 3

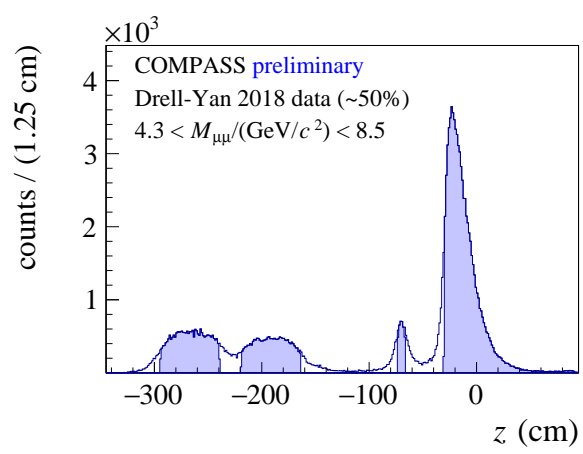

Figure 2: Distribution of dimuon production vertices in the target region. (left top panel). The two-dimensional distribution of the Bjorken scaling variables of pion and nucleon, $x_{\pi}$ and $x_{N}$, for this mass range is presented in Fig. 3 (left bottom panel). The figure demonstrates that the kinematic phase space explored by the COMPASS spectrometer matches the valence region in $x_{\pi}$ and $x_{N}$. In this region, the DY cross section for a proton target is dominated by the contribution of nucleon $u$-quark and pion $\bar{u}$-quark TMD PDFs.

The dimuon transverse momentum $q_{T}$ is required to be above $0.4 \mathrm{GeV} / c$ in order to obtain sufficient resolution in angular variables. The comparison of kinematic dependences of average 
values of different kinematic variables between 2015 and 2018 data is shown in Fig. 3 (left panel). The kinematic dependences in two years are nearly identical.
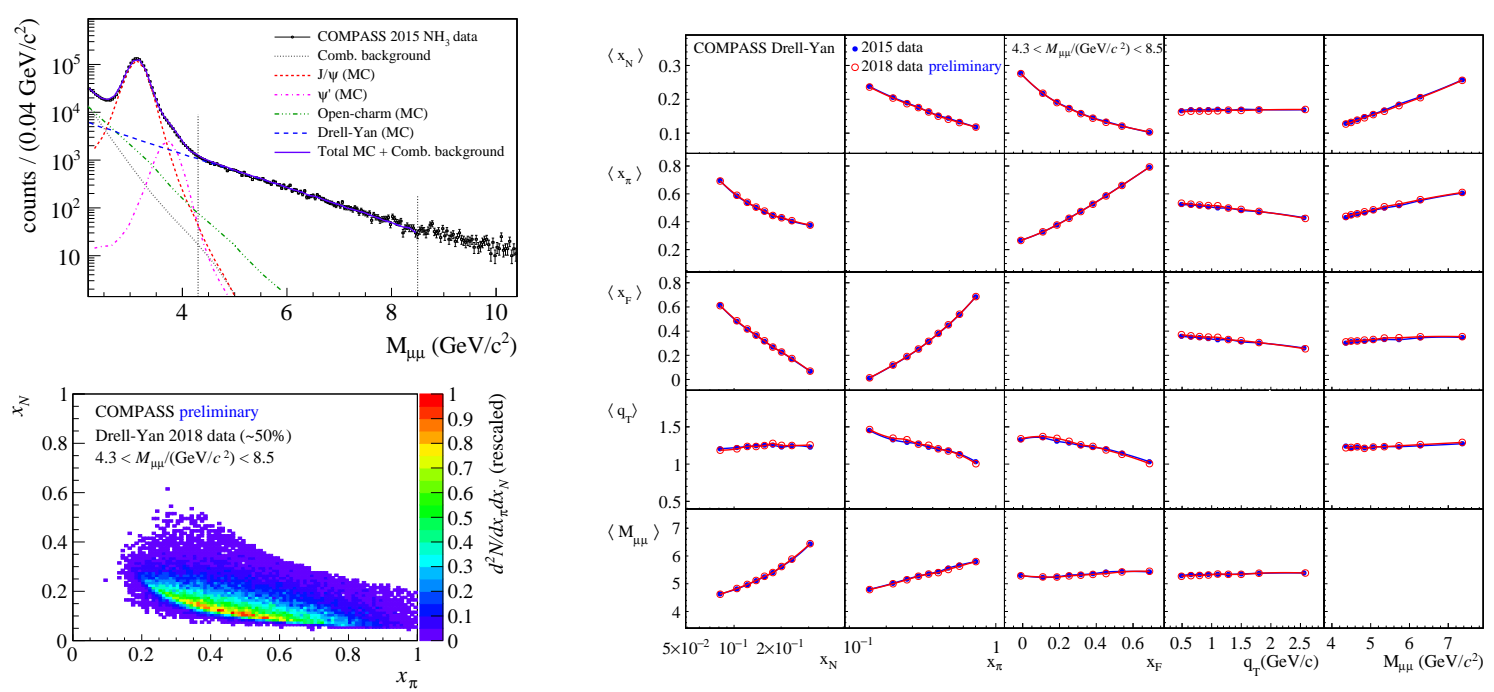

Figure 3: Left top panel: the $M_{\mu \mu}$ distribution. Left bottom panel: the two-dimensional $\left(x_{\pi}, x_{N}\right)$ distribution of the selected high mass dimuons. Right panel: dependences of average kinematic values as extracted from 2015 and 2018 data.

Using an extended Unbinned Maximum Likelihood estimator, all five TSAs are fitted simultaneously together with their correlation matrices. The asymmetries are evaluated in kinematic bins of $x_{N}, x_{\pi}, x_{F}$ or $q_{T}$, while always integrating over all the other variables.

The systematic point-to-point uncertainties are estimated to be about 0.7 times the statistical uncertainties and are indicated in the plots by color bands.

\section{Results and Discussion}

The kinematic dependences for all five Drell-Yan TSAs (twist-2 TSAs, $A_{T}^{\sin \varphi_{S}}, A_{T}^{\sin \left(2 \varphi_{C S}-\varphi_{S}\right)}$ and $A_{T}^{\sin \left(2 \varphi_{C S}+\varphi_{S}\right)}$ and subleading-twist TSAs, $A_{T}^{\sin \left(\varphi_{C S}-\varphi_{S}\right)}$ and $\left.A_{T}^{\sin \left(\varphi_{C S}+\varphi_{S}\right)}\right)$ are shown in Fig. 4 (left panel). Empty circles indicate results obtained from 2015 data, while filled circles correspond to combined 2015 and preliminary 2018 results. Due to the relatively large statistical uncertainties, no clear trend is observed for any of the TSAs.

The rightmost panel in Fig. 4 shows the 2015 measurement and the combined result of 2015 and 2018 for the five extracted TSAs integrated over the entire kinematic range. The preliminary results obtained with part of the 2018 data are found to be in agreement with the published 2015 results [18]. The combined result of 2015 and 2018 for the average Sivers asymmetry $A_{T}^{\sin \varphi_{S}}$ is found to be above zero at about one standard deviation of the total uncertainty. The positive sign and the amplitude of the asymmetry is consistent with the predicted change of sign for the Sivers function. The average value of the TSA $A_{T}^{\sin \left(2 \varphi_{C S}-\varphi_{S}\right)}$ is measured to be below zero with a significance of about two standard deviations. The obtained magnitude of the asymmetry is in agreement with the model calculations of Ref. [19] and can be used to study the universality of the nucleon transversity function. The TSA $A_{T}^{\sin \left(2 \varphi_{C S}+\varphi_{S}\right)}$, which is related to the nucleon pretzelosity TMD 

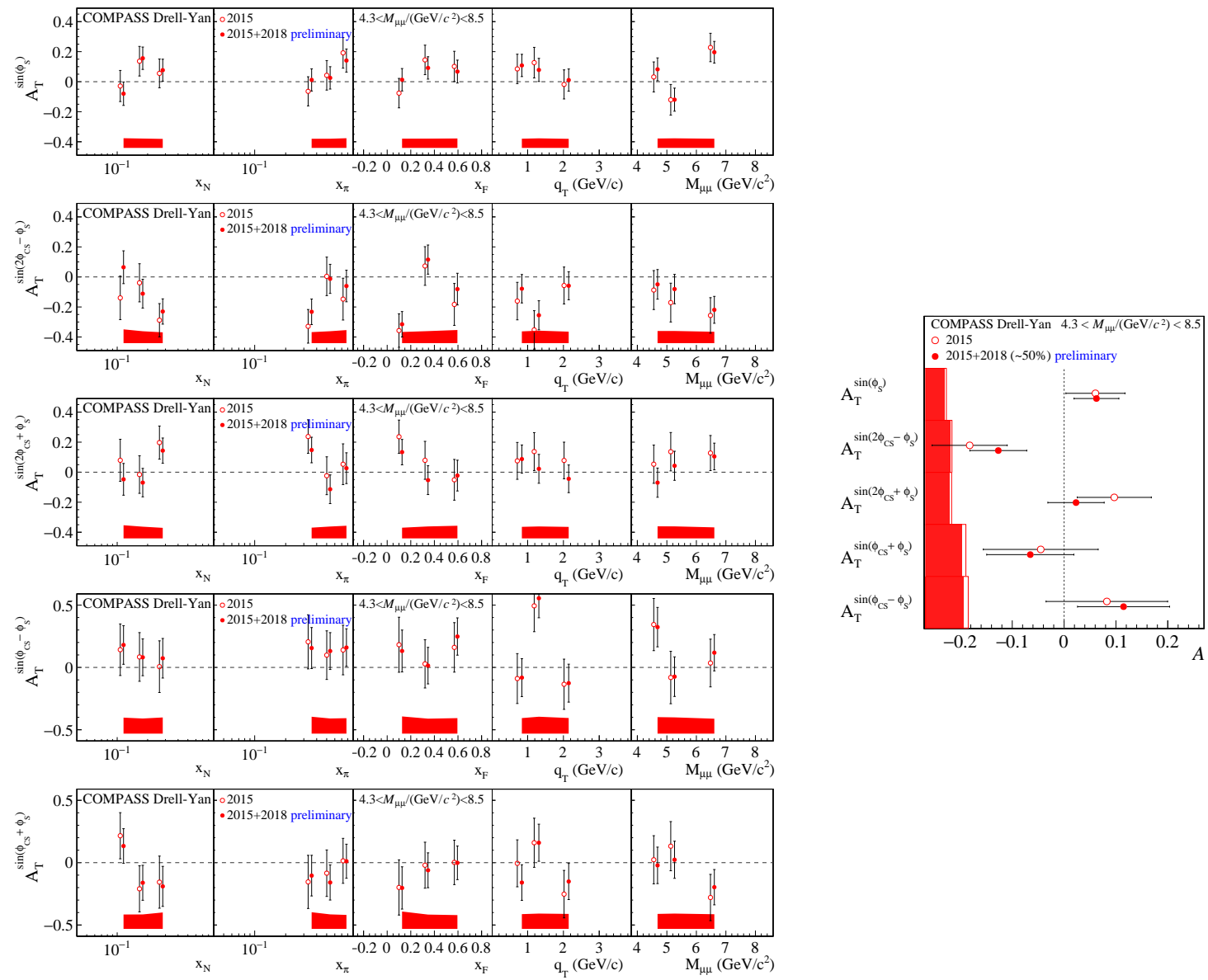

Figure 4: Left panel: Kinematic dependences of extracted Drell-Yan TSAs. Right panel: Drell-Yan TSAs integrated over the entire kinematic range.

PDF, is found to be compatible with zero when combining the results from two years. The remaining two asymmetries are the $A_{T}^{\sin \left(\varphi_{C S}-\varphi_{S}\right)}$ and $\left.A_{T}^{\sin \left(\varphi_{C S}+\varphi_{S}\right)}\right)$ subleading-twist TSAs. Both amplitudes are found to be compatible with zero (see Fig. 4), which can be attributed to the subleading nature of the effects and corresponding dynamic suppressions. The presented results are the first and the only currently available data on the transverse-spin-dependent azimuthal asymmetries in the DrellYan process. COMPASS continues to analyze the data collected in 2018, which will improve the statistical precision of the Sivers and other azimuthal DY TSAs presented in this Letter.

\section{References}

[1] J. Collins, Foundations of perturbative QCD. Cambridge University Press, 2013.

[2] S. Arnold, A. Metz and M. Schlegel, Dilepton production from polarized hadron hadron collisions, Phys. Rev. D79 (2009) 034005, [0 809 .2262].

[3] COMPASS COLlaboration collaboration, F. Gautheron et al., COMPASS-II Proposal, SPSC-P-340, CERN-SPSC-2010-014 (2010) . 
[4] A. Bacchetta, M. Diehl, K. Goeke, A. Metz, P. J. Mulders and M. Schlegel, Semi-inclusive deep inelastic scattering at small transverse momentum, JHEP 02 (2007) 093, [hep-ph / 0611265 ].

[5] A. Kotzinian, New quark distributions and semiinclusive electroproduction on the polarized nucleons, Nucl. Phys. B441 (1995) 234-248, [hep-ph/9412283].

[6] COMPASS COLlaboration collaboration, C. Adolph et al., Experimental investigation of transverse spin asymmetries in muon-proton SIDIS processes: Sivers asymmetries, Phys. Lett. B717 (2012) 383-389, [1205.5122].

[7] COMPASS COLlabORATION collaboration, C. Adolph et al., Experimental investigation of transverse spin asymmetries in muon-p SIDIS processes: Collins asymmetries, Phys. Lett. B717 (2012) 376-382, [1205.5121].

[8] HERMES COllaboration collaboration, A. Airapetian et al., Observation of the Naive-T-odd Sivers Effect in Deep-Inelastic Scattering, Phys. Rev. Lett. 103 (2009) 152002, [0 906 . 3918].

[9] COMPASS collaboration, C. Adolph et al., Sivers asymmetry extracted in SIDIS at the hard scale of the Drell-Yan process at COMPASS, Phys. Lett. B770 (2017) 138-145, [1609. 07374 ].

[10] COMPASS collaboration collaboration, B. Parsamyan, Six “beyond Collins and Sivers” transverse spin asymmetries at COMPASS, Phys. Part. Nucl. 45 (2014) 158-162, [1301. 6615].

[11] D. W. Sivers, Single Spin Production Asymmetries from the Hard Scattering of Point-Like Constituents, Phys. Rev. D41 (1990) 83.

[12] J. C. Collins, Leading twist single transverse-spin asymmetries: Drell-Yan and deep inelastic scattering, Phys. Lett. B536 (2002) 43-48, [hep-ph/ 0204004 ].

[13] Jefferson Lab Hall A collaboration, X. Qian et al., Single Spin Asymmetries in Charged Pion Production from Semi-Inclusive Deep Inelastic Scattering on a Transversely Polarized ${ }^{3}$ He Target, Phys. Rev. Lett. 107 (2011) 072003, [1106.0363].

[14] M. Anselmino, M. Boglione, U. D’Alesio, F. Murgia and A. Prokudin, Study of the sign change of the Sivers function from STAR Collaboration W/Z production data, JHEP 04 (2017) 046, [1612.06413].

[15] M. G. Echevarria, A. Idilbi, Z.-B. Kang and I. Vitev, QCD Evolution of the Sivers Asymmetry, Phys. Rev. D89 (2014) 074013, [1401.5078].

[16] P. Sun and F. Yuan, Transverse momentum dependent evolution: Matching semi-inclusive deep inelastic scattering processes to Drell-Yan and W/Z boson production, Phys. Rev. D88 (2013) 114012 , [1308.5003].

[17] STAR COLLABORATION collaboration, L. Adamczyk et al., Measurement of the transverse single-spin asymmetry in $p^{\uparrow}+p \rightarrow W^{ \pm} / Z^{0}$ at RHIC, Phys. Rev. Lett. 116 (2016) 132301, [1511.06003].

[18] COMPASS collaboration, M. Aghasyan et al., First measurement of transverse-spin-dependent azimuthal asymmetries in the Drell-Yan process, Phys. Rev. Lett. 119 (2017) 112002, [1704.00488].

[19] A. N. Sissakian, O. Yu. Shevchenko, A. P. Nagaitsev and O. N. Ivanov, Polarization effects in Drell-Yan processes, Phys. Part. Nucl. 41 (2010) 64-100. 